\title{
Constructive Approach for Early Extraction of Viral Spreading Social Issues from Twitter
}

\author{
Shiau Chou Jen \\ kyojinsyo@gmail.com \\ The University of Tokyo
}

\author{
Masanao Ochi \\ masanao.oochi@gmail.com \\ The University of Tokyo
}

\author{
Takeshi Sakaki \\ t.sakaki@hottolink.co.jp \\ Hottolink Inc.
}

\author{
Ken Nagahama \\ Kanji Sakai \\ nagahama@dentsu-pr.co.jp \\ kanji@dentsu-pr.co.jp \\ Dentsu Public Relations Inc.
}

\author{
Junichiro Mori \\ Ichiro Sakata \\ mori@mi.u-tokyo.ac.jp \\ isakata@ipr-ctr.t.u-tokyo.ac.jp \\ The University of Tokyo
}

\begin{abstract}
In recent years, there has been a rapid increase in viral spreading social issues that emerge in the public consciousness through the fast spread of information online. With the advent of social media, they sometimes yield unexpected side effects such as product boycotts. Therefore, it is important to recognize them as the earliest and take preventive measures against them. Existing researches on social issue extraction have mainly focused on news channels and newspapers as the primary information sources. However, such approaches cannot be applied for the early extraction of viral spreading social issues because their epicenter is the online public's opinion. In this study, we propose a constructive method inspired by a social issues research approach, called constructivism, for the early extraction of viral spreading social issues. It is characteristic that our method identifies the keywords related to social issues using information obtained from the claims-making activities on Twitter and Twitter-user clustering. We conducted experiments on a large Twitter dataset comprising tens of billions of tweets and the proposed method successfully extracted six out of the seven viral spreading social issues earlier than their first TV news coverage. Furthermore, the proposed method could identify such cases approximately two weeks earlier, on average, than the first national TV news coverage.
\end{abstract}

\section{CCS CONCEPTS}

- Information systems $\rightarrow$ Data extraction and integration.

\section{KEYWORDS}

social issues extraction, viral spreading social issues, Constructivism, Twitter

\section{ACM Reference Format:}

Shiau Chou Jen, Masanao Ochi, Takeshi Sakaki, Ken Nagahama, Kanji Sakai, Junichiro Mori, and Ichiro Sakata. 2020. Constructive Approach for Early Extraction of Viral Spreading Social Issues from Twitter. In 12th ACM

Permission to make digital or hard copies of part or all of this work for personal or classroom use is granted without fee provided that copies are not made or distributed for profit or commercial advantage and that copies bear this notice and the full citation on the first page. Copyrights for third-party components of this work must be honored.

For all other uses, contact the owner/author(s).

WebSci '20, July 6-10, 2020, Southampton, United Kingdom

(c) 2020 Copyright held by the owner/author(s).

ACM ISBN 978-1-4503-7989-2/20/07.

https://doi.org/10.1145/3394231.3397899
Conference on Web Science (WebSci '20), Fuly 6-10, 2020, Southampton, United Kingdom. ACM, New York, NY, USA, 10 pages. https://doi.org/10.1145/ 3394231.3397899

\section{INTRODUCTION}

Solving social issues is indispensable for the sustainable activity of countries and companies. The term "social issues" implies a state that most people find unfavorable [7]. However, social issues can be divided into two categories based on the channel through which they emerge in public consciousness, i.e., "officially announced social issues" and "viral spreading social issues." The first type of social issue is introduced to the public through announcements by government officials and scientific experts; typical examples include climate change and the Sustainable Development Goals. The second type of social issue is introduced to the public through the distribution of information among ordinary citizens.

With the advent of social media in recent years, viral spreading social issues have become more prevalent, and their political and economic impacts are exponentially increasing. "Arab spring" is one of the most influential examples of viral spreading social issues; it was provoked through Facebook [12]. "Ocean plastic pollution" is another influential example of viral spreading social issues; it was recognized by a shocking image of a whale that died from the consumption of plastic bags. Viral spreading social issues quickly spread across communities and yield unexpected side effects, such as sudden fall in stock prices and product boycotts. Therefore, it is important to recognize them in their early stages and take appropriate countermeasures.

In this study, we focus on the early extraction of viral spreading social issues to solve them expeditiously. However, previous methods proposed for the extraction of social issues are not suitable in this case because they only targeted news channels and newspapers $[9,15,31]$. News channels and newspapers are a good sources of information for extracting officially announced social issues because they are the epicenters of those issues. In contrast, the epicenter of viral spreading social issues is public opinion.

To tackle this problem, we propose a constructive approach using Twitter. This approach is inspired by Constructivism, which is a research approach for social issues. In the Constructivism approach, social issues are considered as situations in which the existence of problematic conditions is advocated to other citizens [28]; this is referred to as "claims-making" by constructivists and they examine 
such activities to study social issues. Based on this approach, we used the information relevant to claims-making activities from Twitter and Twitter-user clustering to analyze how these activities are circulated. We chose Twitter as the information source because it is a platform used by many people to comment on daily events and it responds to new problems faster than newspapers and television [3].

Our method consists of four steps. First, we collected tweets in which authors made claims-making activities (called claims-making tweets). To collect such claims-making tweets automatically, we prepared training data whether each tweet is claims-making or not for the first step. Then we built a model that can classify them in the next step accordingly. Then, we used the model to collect claims-making tweets and extracted new social issues from them. In the third step, we performed Twitter-user clustering to analyze the process through which the claims-making activities are spread. In the last step, we extracted words related to new social issues using the $3 \sigma$ burst detection method with the extracted claimsmaking tweets and the clustering result. One of the advantages of the proposed method is its scalability to handle large Twitter data. It is also noteworthy that the proposed method does not require training data to determine whether each word is related to a social issue or not because preparing such training data is difficult for people who are not familiar with a certain social issue [5].

In this study, we collected a large amount of Japanese Twitter data over a period of nine months and performed three experiments. In the first experiment, we observed that our classification model can extract claims-making activities with high precision. In the second experiment, it was observed that the information obtained via claims-making activities and Twitter-user clustering can be used to extract new social issues. In the last experiment, we observed that our method could extract viral spreading social issues earlier than the first TV coverage. The contributions of this study can be summarized as follows.

- To extract viral spreading social issues expeditiously, we proposed a constructive approach to collect and analyze the claims-making activities on Twitter.

- To extract claims-making activities from Twitter, we prepared a new dataset to determine whether each tweet made claims-making and built a high-precision classification model.

- To extract social issues, we proposed a method that does not require training data to determine whether each word is related to social issues and demonstrated that we could extract the viral spreading social issues before they emerged in the public consciousness.

\section{METHOD}

In this section, we describe the four steps that comprise our method. First, we explain how to prepare the training data related to claimsmaking tweets and build a model that can classify them. Then, we explain the Twitter-user clustering method. Subsequently, we explain our method for extracting new social issues from Twitter.

\subsection{Preparing Training Data for Claims-making Tweets}

We used crowdsourcing to prepare the training data for classifying the claims-making tweets based on our previous study [14]. To prepare the training data, we sampled $1,727,787,460$ posts from the Japanese Twitter from December 1, 2018, to August 31, 2019.

First, to enhance the efficiency of crowdsourcing, we excluded tweets that were apparently not claims-making tweets using the following four rules.

(1) Remove tweets that have more than 139 characters.

(2) Remove tweets that do not contain any Japanese characters (hiragana, katakana, or kanji).

(3) Remove tweets containing emojis.

(4) Remove tweets from bots and the official accounts of organizations.

We introduced Rule (1) because tweets with more than 139 characters are omitted by the official Twitter API. Owing to the nature of Japanese syntax, the meaning of the whole sentence cannot be understood unless the end of sentence is known. Rule (2) was introduced because we focused on the Japanese viral spreading social issues. Rule (3) was adopted because it can be inferred that tweets containing emojis tend to be positive topics. Rule (4) was applied to remove posts by bot accounts and campaign tweets. By implementing these rules, the number of tweets was roughly reduced by half, thereby doubling the efficiency of crowdsourcing. Furthermore, we applied the following pre-processing rules to the tweets before submitting the tasks to the annotators.

(1) Remove linefeed codes such as $\backslash n$ and $\backslash r \backslash n$.

(2) Convert strings starting with "http" or "https" into "URL."

(3) Replace strings consisting of "@" and account name with "@USER."

(4) Replace \&amp, \&gt; , and \&lt; with \&, >, and <, respectively.

We used Yahoo! crowdsourcing ${ }^{1}$ as the crowdsourcing platform. We did not use the term "claims-making activity" for our task description because the annotators are usually unfamiliar with the technical term of constructivism. Instead, we submitted tasks that asked people to determine whether the author of a tweet seemed to criticize something or raised an issue. We sampled 68,634 tweets and separated them into 38,646 and 29,988 tweets owing to the limitations of the platform. We assigned seven annotators to one tweet. The task limit time was set to $15 \mathrm{~min}$. to ensure that the annotators had sufficient time to complete their task. Each annotator was assigned 36 questions. Nine of the 36 question were "check questions."; check questions are questions that are assigned ground truth label in advance by the task owner. With the help of check questions, we successfully excluded the annotations by the annotators who could not answer all check questions correctly. Table 1 presents examples of the check questions. The first example is negative in sentiment but not a claims-making tweet because it lacks a target of criticism target. However, the last example is a claims-making tweet because it criticizes the violence inflicted on a member of a Japanese idol group.

\footnotetext{
${ }^{1}$ https://crowdsourcing.yahoo.co.jp/
} 
Table 1: Examples of check questions. Note that these tweets were translated from Japanese to English.

\begin{tabular}{c|c}
\hline tweet & correct label \\
\hline \hline I'm very tired... and I have a headache. & not claims-making \\
\hline $\begin{array}{c}\text { It was very delicious } \\
\text { \#golden five noodle URL }\end{array}$ & not claims-making \\
\hline Why do these parents abuse their child? & claims-making \\
\hline $\begin{array}{c}\text { RT @ USER I've decided not to comment } \\
\text { on entertainment too much, but } \\
\text { I can't help talking about NGT. }\end{array}$ & claims-making \\
\hline
\end{tabular}

\subsection{Building Classification Model}

We developed a model that can classify whether a tweet is a claimsmaking tweet using the annotated data prepared in Section 2.1 to extract the claims-making tweets automatically from Twitter.

First, we designed the features of the classification model. There are several feature designs for tweet classification, such as $\mathrm{N}$-gram [24], part-of-speech [23], hashtags and lexical features [1]. In recent years, word embedding such as Word2Vec [21] have been widely used as features. Therefore, we also used a type of word embedding called hottoSNS [20]; it is a word embedding pre-trained by a largescale Japanese SNS including Twitter and Web corpus. We chose hottoSNS because unlike other pre-trained word embedding, it is trained by a corpus similar to our task.

Subsequently, we selected the classification models. Various models have been proposed by previous studies to classify tweets, such as Naive Bayes [27], support vector machine (SVM) [2], convolutional neural networks (CNNs) [29], and long short-term memory (LSTM) [11]. Kim reported that CNNs exhibit remarkable performance for text classification [16]. Wang et al. showed that the LSTM [11] based on word embedding was superior to the maximum entropy model and SVM [32]. From the models proposed by previous studies, we selected and built four models, namely logistic regression (LR), multi-layer perceptron (MLP), gradient boosting tree (GBT), and CNNs. We did not use LSTM because it requires a significantly longer training time in comparison to other models. Instead of LSTM, we selected CNN because it can capture the context information of a tweet as efficiently as LSTM, but it requires less training time. LR and MLP are the baseline models. GBT is the strong baseline model, which has been shown to yield state-of-the-art results on many standard classification benchmarks [4].

Before training each model, we tokenized each tweet using $\mathrm{MeCab}^{2}$, a Japanese tokenizer, with a NEologd dictionary ${ }^{3}$. We represented a tweet as a 200-dimensional vector that denoted the sum of the word vectors in the tweet. We employed the vector representation of a tweet as a feature for LR, MLP, and GBT. If a word was not registered in hottoSNS, its corresponding embedding was considered as a 200 -dimensional 0 vector. For the $\mathrm{CNN}$ model, we used a $200 \times 80$-dimensional tensor aligned with each word's

\footnotetext{
${ }^{2}$ http://taku910.github.io/mecab/

${ }^{3}$ https://github.com/neologd/mecab-ipadic-neologd
}

embedding. We only used the first 80 words of the tweets that contained more than 80 words. If a tweet had less than 80 words, we padded the trailing elements as 200 -dimensional 0 vectors.

Claims-making tweets were grossly fewer than non-claims-making tweets, which gave rise to the challenge of highly imbalanced data. To deal with this challenge, we adjusted a loss function for our optimization to determine the parameters $W$ of the models. We used the weighted cross-entropy expressed by Eq. (1) as our loss function.

$$
E(W)=-\sum_{n=1}^{N} \frac{y_{n} \log p_{n}}{N_{\text {positive }}}+\frac{\left(1-y_{n}\right) \log \left(1-p_{n}\right)}{N_{\text {negative }}}
$$

$W$ was determined by the gradient descent method. In Eq. (1), $y_{n}$ is the ground-truth label for the $n$th sample, $p_{n}$ is the model's predicted probability of whether the $n$th sample represents a claimmaking tweet, and $N_{\text {positive }}$ and $N_{\text {negative }}$ denote the numbers of claims-making and non-claims-making tweets in the training data, respectively. After calculating $W$, we compared the performance of each model on the test data and determined the model. Then, we deployed the best model for extracting the claims-making tweets from the Twitter data.

\section{$2.3 \quad$ Twitter-User Clustering}

In this step, we performed user clustering to analyze whether a claims-making tweet circulated across Twitter. To perform user clustering, we first extracted the text information of each user's Twitter profile because it included information about the user's affiliations and interests. Accordingly, we obtained a vector representation of each user's profile data.

Our clustering method included the following steps. First, we implemented a morphological analysis on each profile text using MeCab with the NEologd dictionary. Then, we extracted the nouns and proper nouns from each profile and weighted them based on their TFIDF value. The TFIDF value of a word $w$ in the profile of a user $u$ is defined by Eq. (2).

$$
\operatorname{TFIDF}_{w, u}=\frac{n_{w, u}}{\sum_{w \in u} n_{w, u}} \times \log \frac{|U|}{|u: u \ni w|}
$$

$n_{w, u}$ denotes the frequency of appearance of the word $w$ in the profile of user $u .|U|$ is the total number of users and $|u: u \ni w|$ indicates the number of the users' profile that contains the word $w$. After weighting each word, we extracted the top three words as the representative words for each user, and converted them into 200-dimensional vectors using hottoSNS. Finally, each user's vector representation was obtained by averaging the top three word vectors. We utilized the k-means clustering algorithm for the obtained vector representations and split the Twitter users into 20 clusters. The number of clusters was determined heuristically.

\subsection{Social Issue-related Word Extraction}

In the final step, we extracted the keywords related to new social issues. Viral spreading social issues rapidly emerge in the public consciousness after being propagated by an individual. In this process, the number of claims-making tweets mentioning an issue rapidly increase, and consequently, words representing the issue appear more frequently than usual. Exploiting this phenomenon, 
Figure 1: Overview images of our extraction method for new social issues. The first image shows the daily frequency of a word in the claims-making tweets and the second image shows the cluster entropy of a word defined by Eq.(3)

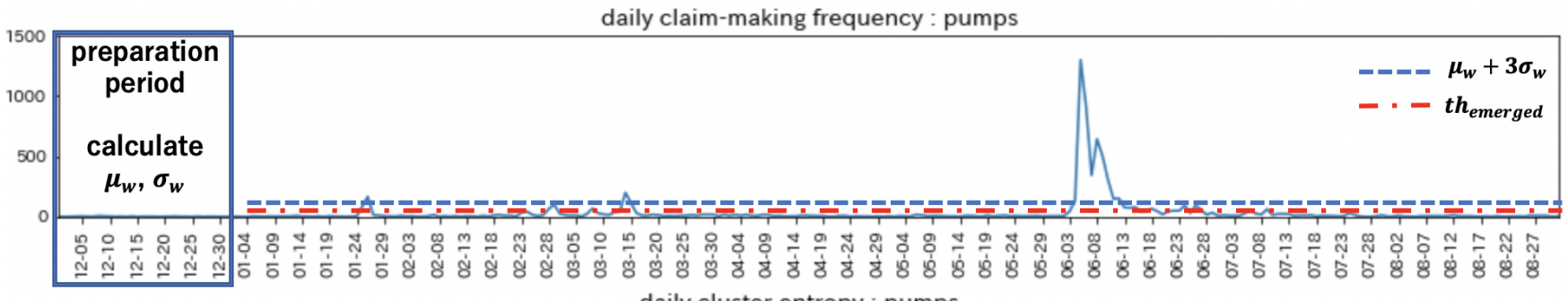

daily cluster entropy : pumps

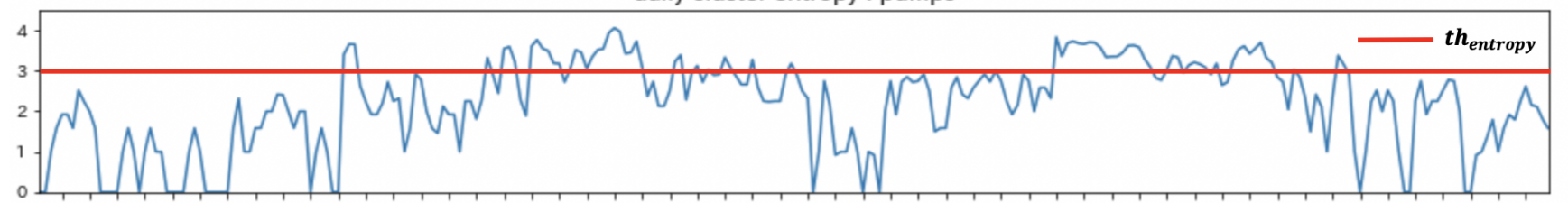

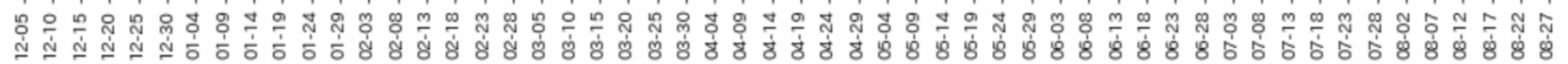

we attempted to extract new viral spreading social issues through the burst detection method $[8,10,17]$. In this study, we used a large amount of Twitter data (more than 5 million tweets per day); therefore, we propose a computationally efficient method based on the $3 \sigma$ burst detection method. $3 \sigma$ method is computationally cheap because it only requires the average and standard deviations of the target distribution. Mizunuma et al. also used the $3 \sigma$ method to capture the burst phenomenon [22]. However, they computed a common threshold for all words to determine the burst state, which can lead to the burial of new social issues under other topics, as is often the case with TV programs and commercial campaigns. Therefore, unlike Mizunuma et al., we computed the threshold for each word differently to tackle the new social issues separately from the popular topics.

Our method determined whether each word $w$ underwent bursting at each period $t$. Figure 1 presents an overview of the proposed method. In our method, the frequency of word $w$ in the extracted claims-making tweets is referred to as the claims-making frequency, which is denoted by $f_{r e q}, t$. First, we computed the average of each word $\mu_{w}$ and the standard deviation $\sigma_{w}$ of the claims-making frequency during period before we started the extraction process (called preparation period). Then, we considered that each word underwent bursting if both $f r e q_{w, t}$ and $f r e q_{w, t-1}$ exceeded $\mu_{w}+3 \sigma_{w}$. Such words were considered as the candidates for new social issues at period $t$. To extract new social issues, we removed the words whose maximum claims-making frequency was larger than $t h_{\text {emerged }}$ during the preparation period. $t h_{\text {emerged }}$ was set to $\mu+3 \sigma . \mu$ denotes the average of the claims-making frequency of all words during the preparation period and $\sigma$ denotes its standard deviation. Furthermore, it should be noted that words which rarely appeared during the preparation period may become noise because the value of $\mu_{w}+3 \sigma_{w}$ for such words was extremely small. To filter such words, we used $t h_{\text {emerged }}$ as the threshold, and removed the words whose $f_{r e q}, t+f_{r e q}, t-1$ values were less than $t h_{\text {emerged }}$ from the new social issues candidates.
In addition to the $3 \sigma$ method for examining the claims-making frequency, we considered the diversity of the keyword used among the clusters of users because social issues are public issues that should naturally spread across many clusters. To measure the extent of spread at every period $t$, we computed the entropy of claimsmaking frequency $H_{w, t}$, defined by Eq.(3).

$$
H_{w, t}=\sum_{c=1}^{|C|}-p_{w, t}^{c} \log p_{w, t}^{c}
$$

Here, $c$ indicates the cluster number and $|C|$ is the total number of clusters. $p_{w, t}^{c}$ denotes the claims-making frequency of Cluster $c$ divided by the population of the cluster. A lower $H_{w, t}$ indicates that the word appears only in certain clusters. After removing the words word whose $H_{w, t}$ values were lower than threshold $t h_{\text {entropy }}$ from the social issues candidates, we finally obtained words that were related to new social issues at period $t$.

\section{EXPERIMENTS AND RESULTS}

In this section, we first describe the data used in the experiments, followed by the setteings of the three experiment and the results.

\subsection{Dataset}

For the evaluation experiments, we sampled 1,727,787,460 Japanese tweets from December 1, 2018, to August 31, 2019. We used these data to create a training dataset for building a classification model and extracted the claims-making tweets. We also obtained 24 million user profile descriptions from December 1, 2018, to August 31, 2019, using the official Twitter $\mathrm{API}^{4}$ and excluded the users who had less than two nouns or proper nouns in their profiles. We used these data for Twitter-user clustering. Hottolink, Inc. supported us in the collection of Twitter data. We also prepared data collected from Nikkei newspaper articles and TV programs for evaluation.

${ }^{4}$ https://help.twitter.com/ja/rules-and-policies/twitter-api 
NikkeiTelecom ${ }^{5}$ and MetaTV ${ }^{6}$ were used to obtain data related to newspaper articles and TV programs, respectively. These data were collected from January 1, 2018, to December 31, 2019.

\subsection{Experiment 1: Claims-making Tweets Classification}

Experiment 1 aimed to determine the possibility of extracting claims-making activities from Twitter. To confirm this, we evaluated the classification performances of the models described in Section 2.2.

First, we created training data from the annotated results. The crowdsourced data may contain noise; therefore, we used Krippendorf's $\alpha$ to obtain reliable training data. Krippendorf's $\alpha$ measures the inter-annotator agreement; $\alpha$ is ranges from [0-1], and a higher $\alpha$ corresponds to better training data. If the value is more than 0.67 , the quality of training data is enough for research usage. We separated the annotated results into positive and negative examples, according to the number of annotators who classified a tweet to be a claims-making tweet. For the first annotation result, we used tweets that were considered to be claims-making tweets by more than three annotators as positive examples, and the tweets that were not considered to be claims-making tweets by anybody were used as negative examples. Krippendorf's $\alpha$ for this separation was measured to be 0.855 . For the second annotation result, we used tweets that were considered to be claims-making tweets by more than four annotators as positive examples, and tweets that were not considered to be claims-making tweets by anybody were used as negative examples. Krippendorf's $\alpha$ for this separation was measured to be 0.782 . After data separation, we obtained a dataset consisting of 6586 positive tweets (claims-making tweets) and 37578 negative tweets (non-claims-making tweets).

We split the obtained data randomly into training, validation, and test data for model evaluation. Each data type contained $80 \%$, $10 \%$, and $10 \%$ of the dataset, respectively. Training data were used to determine the value of parameter $W$ of each model. Validation data were used to implement early stopping and hyperparameter tuning. Test data were used to evaluate the performance of the models. We used AUC as an evaluation metric because other metrics, such as precision, recall, and $\mathrm{f} 1$, depend on the predicted probability threshold, which determines the positive and negative labels.

We used the scikit-learn $\mathrm{API}^{7}$ for the implementation of LR and $\operatorname{lightgbm}^{8}$ for the implementation of GBT. MLP and CNN were built using PyTorch ${ }^{9}$. As a hyperparameter for LR, the value of the 12 regularization coefficient was set to 10 . As hyperparameters for the GBT, the number of boosting stages, maximum depth of the individual estimators, learning rate, and early stopping rounds were set to be $4000,8,0.01$, and 40 , respectively. As hyperparameters for MLP, the batch size, number of epochs, number of layers, dimension size of the first layer, dimension size of the second layer, learning rate, and early stopping rounds were set to be 256, 300, 2, 150, 100, 0.01 and 40, respectively; additionally, Adam and ReduceLROnPlateau were selected as the optimization algorithm and

\footnotetext{
${ }^{5}$ http://telecom.nikkei.co.jp/

${ }^{6}$ https://www.metatv.jp/

${ }^{7}$ https://scikit-learn.org/stable/

${ }^{8}$ https://lightgbm.readthedocs.io/en/latest/index.html

${ }^{9}$ https://pytorch.org/
}

Table 2: Results of claims-making tweet classification. CNN outperformed LR and MLP; it also outperformed the strong baseline, i.e, GBT.

\begin{tabular}{c|cccc}
\hline Model name & LR & GBT & ML & CNN \\
\hline \hline AUC & 0.944 & 0.959 & 0.955 & $\mathbf{0 . 9 6 1}$ \\
\hline
\end{tabular}

Table 3: Extraction performance comparison before and after threshold optimization. We used an optimized threshold to extract claims-making tweets.

\begin{tabular}{c|cccc}
\hline threshold & f1 & precision & recall & extracted number \\
\hline \hline 0.5 & 0.668 & $\mathbf{0 . 8 9 2}$ & 0.535 & $46,786,826$ \\
\hline 0.2 (optimized) & $\mathbf{0 . 7 3 7}$ & 0.697 & $\mathbf{0 . 7 8 2}$ & $\mathbf{6 8 , 3 8 7 , 4 7 3}$ \\
\hline
\end{tabular}

learning rate scheduler, respectively. As hyperparameters for $\mathrm{CNN}$, the batch size, the number of the epoch, number of filters, filter sizes, learning rate, and early stopping rounds were set to be 256, 300, 54 [(2,200), (3,200), (4,200)], 0.01, and 40, respectively; Adam and ReduceLROnPlateau were used as the optimization algorithm and learning rate scheduler, respectively. We determined these hyperparameters according to the performance of the models on the validation data.

\subsection{Result of Experiment 1: Claims-making Tweet Classification}

Table 2 summarizes the results of claims-making tweet classification. The CNN achieved the highest AUC, 0.961, and it outperformed the performances of LR and MLP. It was also observed that the CNN outperformed a strong baseline, GBT. Compared to other models, the $\mathrm{CNN}$ could capture the context information of tweets; therefore, this result was reasonable.

Then, we deployed the CNN model to extract the claims-making tweets from the entire Twitter data. In general, tweets with a predicted probability of 0.5 or more are considered to be positive examples. If a higher threshold is selected, we can extract higher quality claims-making tweets; however the number of claims-making tweets will decrease. In this study, we optimized the probability threshold to maximize the $\mathrm{f} 1$ value using the validation data. Table 3 presents the result of test data after threshold optimization. Although the precision for the positive examples dropped from 0.892 to 0.697 , the $\mathrm{f} 1$ value increased from 0.668 to 0.737 and the recall increased from 0.535 to 0.782 . This implies that our model can extract nearly $80 \%$ of the entire claims-making tweets, and approximately $70 \%$ of them were actual claims-making tweets. Using this threshold, we extracted 68,387,473 claims-making tweets from a total of $1,727,787,460$ tweets. Therefore, it can be concluded that the claims-making activities can be extracted from Twitter.

\subsection{Experiment 2: Social Issue-related Word Extraction}

Experiment 2 aimed to examine whether the identification of claimsmaking activities via tweet classification and their spreading process determined via Twitter-user clustering make any contribution to the extraction of social issues. To confirm this, we compared the 
Table 4: Examples of correct social issue-related words for the social issue extraction method. This list includes several words related to social issues such as, "elderly driver" and "bullying problem," but some words are noise, such as "turning point" and "sink."

Education Agency, fight, drive recorder, social experiment, respect for basic human rights, economic policy, weakness, sink, buyer, biodiversity, Japan Post Insurance, special mission, summit, elderly driver, raswell, surname, hat, ingenuity, farm, turning point, bullying problem, government office, poor

performance of the proposed method with that of other methods, such as the method that uses whole tweets, that without Twitteruser clustering, and that randomly extracts 10,000 words.

To evaluate the performance of each method, we created a list of social issue-related words from articles obtained from the Nikkei newspaper from January 1, 2019, to August 31, 2019. This list of words was referred to as the correct word list. It was created by the following procedure. First, we extracted news articles with themes defined as social issues and excluded those that did not contain the word "issue." Then, we extracted sentences containing words such as "issue," "dispute," "controversy" from the news articles. Subsequently, we compared the frequency of each common noun and proper noun in 2018 and 2019 articles, respectively. Finally, we defined words that appeared more frequently in 2019 than, as correct words related to new social issues. Following these steps, we obtained 4064 words that constituted the correct word list.

Table 4 lists examples of words from the list. It can be observed that many social issue-related words are included in the list. For example, "elderly driver" is a term related to the problem of dangerous elderly driving in Japan, "Japan Post Insurance" is a term related to the problem of illegal sales of Japan Post Insurance, and "drive recorder" is a term related to the problem of tailgating in Japan. In contrast, it contains a few words that are unlikely to be related to social issues, such as "turning point" and "sink."

We only extracted the common nouns and proper nouns by our methods because the correct word list only contains common nouns and proper nouns. Each model's performance was evaluated using precision, recall, and $\mathrm{f} 1$ as the evaluation metrics in relation to the correct word list. As a hyperparameter for the proposed method, the value of $t h_{\text {entropy }}$ was set to be 3 ; the same value was used for the method that used whole tweets.

\subsection{Result of Experiment 2: Social Issue-related Words Extraction}

In this section, we first present the results of Twitter-user clustering. Then, we present the results of social issue-related word extraction.

Table 5 presents the results of Twitter-user clustering as well as the representative words of each cluster. The representative words are the top five TFIDF words among the clusters. We also manually added an explanation label for each cluster. It can be observed that we successfully classified Twitter users according to their interests. For example, Clusters 0 and 10 represent the clusters of people who like consumer games or shooting games. However
Table 5: Result of Twitter-user clustering. Representative words are top five TFIDF words among each cluster, and we manually added explanation labels to each cluster. It can be observed that Twitter users are separated by their interests.

\begin{tabular}{|c|c|c|}
\hline cluster & annotated label & representative words \\
\hline 0 & consumer game & $\begin{array}{c}\text { game, Pokémon, TRPG, } \\
\text { Game commentary, Rhythm game }\end{array}$ \\
\hline 1 & adult & $\begin{array}{l}\text { ill acount, sex friend, } \\
\text { offline sex, girl, mentally ill }\end{array}$ \\
\hline 2 & anime song & LiSA, crew, utaite, live, band \\
\hline 3 & Japanese idol & $\begin{array}{l}\text { Nogizaka, Say, SMAP, } \\
\text { NEWS, Keyakizaka46 }\end{array}$ \\
\hline 4 & bike rider & bike, bicycle, road bike, camera, rider \\
\hline 5 & sports freak & soccer, fan, Carp, Hawks, basket ball \\
\hline 6 & anime idol & $\begin{array}{l}\text { Aqours, Uta no pricess, } \\
\text { Idolish } 7 \text {, hako-oshi, lovelive }\end{array}$ \\
\hline 7 & foreigners & you, baby, and, life, the, all, reprint \\
\hline 8 & smartphone game & $\begin{array}{l}\text { Monster Strike, Pazzle \& Dragons, } \\
\text { rank, Band Dream, Shadow Bath }\end{array}$ \\
\hline 9 & adult male & ticket, ramen, event, goods, Nagoya \\
\hline 10 & shooting game & Splatoon, Fortnite, game, PUBG, Siege \\
\hline 11 & Disney & doll, Disney, rabbit, illustration, goods \\
\hline 12 & net geek & $\begin{array}{l}\text { cryptocurrency, blog, engeneer, } \\
\text { buisiness, } 2 \text { mellion }\end{array}$ \\
\hline 13 & right wing & $\begin{array}{l}\text { Japanese people, Copyright, } \\
\text { musical, Japanese, drama }\end{array}$ \\
\hline 14 & ordinary people & $\begin{array}{l}\text { follow-back, NaNa, tweet, } \\
\text { ill account, mutual follow }\end{array}$ \\
\hline 15 & $\begin{array}{l}\text { junior and senior } \\
\text { high school students }\end{array}$ & $\begin{array}{c}\text { baseball club, soccer club, } \\
\text { basketball club, soft, valley club }\end{array}$ \\
\hline 16 & K-POP & TWICE, EXO, ARMY, once, SHINee \\
\hline 17 & AR smartphone game & compass, character, Pokémon, guild, deck \\
\hline 18 & $\mathrm{~J}-\mathrm{POP}$ & GREEN, King, Happiness, Order, Reprint \\
\hline 19 & anime freak & $\begin{array}{l}\text { anime, manga, Vocaloid, } \\
\text { voice actor, young adults books }\end{array}$ \\
\hline
\end{tabular}

Cluster 3 represents the cluster of people who like idol groups and Cluster 6 represents users who like idol groups in anime. We can also observe the clusters of people who like anime songs (Cluster 2), JPOP(Cluster 18), KPOP(Cluster 16).

Table 6 summarizes the results of social issue-related word extraction. We compared the performances of the methods that used whole tweets and claims-making tweets; moreover, we compared the performance of both methods with and without cluster entropy. The proposed method yielded a significantly better $\mathrm{f} 1$ value, 0.176 , than the method that used whole tweets, 0.129. It is evident that the cluster entropy increased the precision from 0.105 to 0.113 for the method that used whole tweets; it also increased the precision of the method that used claims-making tweets from 0.113 to 0.126 . Thus, the results demonstrate that the identification of claimsmaking tweets and Twitter-user clusters are valid approaches for the extraction of social issues.

Table 7 shows sample words extracted by each method. It can be observed several social issue-related words were identified by the proposed method, such as "Hong Kong" (the Hong Kong protest), "Akie Abe" (wife of Japanese prime minister who was criticized by opposition party), and "spa!"(Japanese magazine published a controversial article insulting female students). The other method also extracted several social issue-related words, such as "amendment, Constitution" (the Japanese government's attempt to amend the constitution was controversial) and "Aichi prefecture", "Daisuke Tsuda" (he was an artistic director of the art event Aichi Triennale). However, thus method also extracted unsuitable words, such as 
Table 6: Results of social issue-related words extraction. The ${ }^{*}$ denotes the proposed method; it yields the highest $\mathrm{f} 1$ value.

\begin{tabular}{c|ccc}
\hline method & precision & recall & f1 \\
\hline random extractor & 0.014 & 0.035 & 0.02 \\
whole tweets & 0.105 & 0.155 & 0.126 \\
whole tweets + cluster & 0.113 & 0.152 & 0.129 \\
claims-making tweets & 0.113 & $\mathbf{0 . 3 2 1}$ & 0.167 \\
claims-making tweets + cluster & $\mathbf{0 . 1 2 6}$ & 0.292 & $\mathbf{0 . 1 7 6}$ \\
\hline
\end{tabular}

Table 7: Word examples extracted by each method. The * denotes the proposed method. The proposed method could extract many social issue-related words, while the number of unsuitable words is few.

\begin{tabular}{cc}
\hline using whole tweets & using claims-making tweets * \\
\hline amendment, Koicha & ivory, Hong Kong, security, \\
Haruka Amami, Constitution & Akie Abe, sexual harassment \\
Aichi prefecture, Kurazushi, & JASRAC, unpaid, Bulgaria \\
apology interview, G7, & Discrimination against women, \\
Daisuke Tsuda, Kaneka & 200 times, U.S. Forces in Japan \\
heavy rain, TV anime & tapioca, spa!, pineapple \\
brother, Chrono Trigger, & Taiwan, green juice \\
\hline
\end{tabular}

anime character name (Haruka Amami), product name (Koicha), and game title (Chrono Trigger). This is because whole tweets contain both claims-making tweets and non-claims-making tweets.

\subsection{Experiment 3: Early Extraction of Viral Spreading Social Issues}

Experiment 3 is the primary experiment of this study wherein we investigated whether viral spreading social issues can be extracted before they emerge in the public consciousness. We extracted new social issues similar to Experiment 2 and compared the day when the issues were extracted by our method and when they were recognized by the public.

To determine our target viral spreading social issues, we investigated social issues featured by the Nikkei Newspaper article and classified each issue as viral spreading or officially announced. Subsequently, we determined the target extraction words for each issue based on the words used in the articles. Dentsu Public Relations Inc. supported this process. Table 8 presents examples of our target viral spreading social issues, target words, and descriptions of each issue. We consider the day when target words were extracted as the day when the issue was extracted.

As a day when ordinary citizens recognize each issues, we investigated when these seven issues were broadcasted on the six most popular TV stations (NHK, Japan TV, TBS, Fuji TV, TV Asahi, TV Tokyo) using MetaTV. Dentsu Public Relations Inc. also supported this investigation. In this study, we used four different criteria to determine public recognition; i.e., $T V_{\text {first }}, T V_{10 m}, N H K_{\text {first }}$ and $N H K_{5 m}$, because the degree of recognition is correlated with the TV stations that broadcast an issue and the amount of exposure received by a particular issue. The first criterion $T V_{\text {first }}$ represents the day when an issue was first broadcasted in either of the six
TV stations. This criterion indicates the day when relevant information began to reach many citizens. The second criterion $T V_{10 m}$ represents the day when the six TV stations broadcasted an issue for a total of $10 \mathrm{~min}$ or more. This day implies that the issue has become serious. The third criterion $N H K_{\text {first }}$ represents the day when an issue was first broadcasted on NHK. We adopted this criterion because NHK is considered to be a more trustworthy TV station than others [13]. The last criterion $\mathrm{NHK}_{5 m}$ represents the day when NHK broadcasted an issue for more than $5 \mathrm{~min}$.

Using these criteria, we evaluated the number of issues that can be extracted in advance and the average lead time. While calculating the average lead time, we excluded the maximum and minimum lead time to filter the outliers, and defined the lead time of the issues that cannot be extracted using our method as 0 days.

\subsection{Result of Experiment 3: Early Extraction of Viral Spreading Social Issues}

Table 9 summarizes the results of the early extraction performed for the seven target viral spreading social issues. We successfully extracted almost all the target issues earlier than the first TV coverage, except the issue of "Image download illegalization." Specifically, we extracted the issue "KuToo" one month earlier than $T V_{\text {first }}$ and approximately four months earlier than $\mathrm{NHK}_{5 m}$. We could not extract the issue "Image download illegalization" earlier than $T V_{\text {first }}$ and $N H K_{\text {first }}$; however, we were able to extract it earlier than $T V_{10 m}$ and $N H K_{5 m}$. In the last row of Table 9, we show the average lead time according to each criteria. On average, our method could extract viral spreading social issues 3.2 days earlier than the first TV coverage and two weeks earlier than the first national TV news coverage. Therefore, we can conclude that our method can extract viral spreading social issues in their initial stages.

\section{DISCUSSION}

In this section, we discuss three typical issues, i.e., "KuToo" as an example of civic movements, "cherry blossom party" as an example of political issues, and "Image download illegalization" as an example of the failed case. Then, we explain the application of this study and limitations of our method.

"KuToo" was triggered by a tweet on January $24^{10}$ and a petition to stop this issue started on March 12; the petition was submitted to the Ministry of Health, Labor and Welfare on June $3^{11}$. Considering this background, we can conclude that the proposed method could extract "KuToo" issue in its early stage. The cumulative claimsmaking frequency of the term "KuToo" is shown in Fig. 2 (a). As shown in the figure, the word appeared on January 24; subsequently, a sudden increase in its claims-making frequency was observed. A significant increase was observed on June 3 owing to the submission of petition signatures. It is characteristic for civic movements to observe a considerable time delay between the beginning and the day when the movement emerges in the public consciousness. We can also see the clusters that actively reacted to this issue; Cluster 13 (right wing), followed by Cluster 12 (net geek).

"Cherry blossom party" is an issue related to the 64th cherry blossom party that was held on April 13. One TV station reported

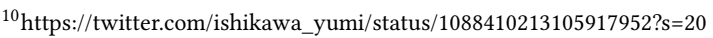

${ }^{11} \mathrm{https}$ //time.com/5548873/japan-kutoo-high-heels-metoo/
} 
Table 8: Target viral spreading social issues and target words for each issue. Note that issue names and target words are translated from Japanese to English.

\begin{tabular}{|c|c|c|}
\hline issues name & target words & issues description \\
\hline KuToo & KuToo, pumps & $\begin{array}{l}\text { A woman criticized the working style that female workers are } \\
\text { required to wear pumps and launched a petition to stop such style. }\end{array}$ \\
\hline Cherry blossom party & Cherry blossom party & $\begin{array}{l}\text { Japanese prime minister was suspected that he invited unsuitable } \\
\text { persons to the official cherry blossom party. }\end{array}$ \\
\hline Image download illegalization & Image download illegalization & $\begin{array}{l}\text { It was considered to ban downloading an image contains authorized } \\
\text { content. But it was withdrawn due to the severe opposition }\end{array}$ \\
\hline Part-timer's prank videos & $\begin{array}{l}\text { part-timer's prank videos, } \\
\text { Sukiya, KuraZushi }\end{array}$ & $\begin{array}{l}\text { Part-timers at Sukiya and Kurazushi published prank videos on SNS, } \\
\text { and the videos caused enormous economic damage to the companies. }\end{array}$ \\
\hline SPA! controversial article & spa, spa! & $\begin{array}{l}\text { A magazine SPA! published a ranking on } \\
\text { which female university is seductive. }\end{array}$ \\
\hline Kaneka paternity harassment & Kaneka & $\begin{array}{l}\text { A Kaneka male employee who just returned from childcare leave } \\
\text { was ordered to transfer a distant place. }\end{array}$ \\
\hline Aichi Triennale & Aichi Triennale & $\begin{array}{l}\text { Excessively political exhibits at the art festival called Aichi Triennale } \\
\text { was suspended by the prefectural government. }\end{array}$ \\
\hline
\end{tabular}

Table 9: Results of the early extraction of viral spreading social issues. The proposed method could extract almost all issues earlier than their first TV coverage, except one issue. On average, we could extract viral spreading social issues 3.2 days earlier than the first TV coverage and 14 days earlier than the first national TV coverage.

\begin{tabular}{|c|c|c|c|c|c|}
\hline issues name & $\begin{array}{c}\text { extracted } \\
\text { day }\end{array}$ & $\begin{array}{c}T V_{\text {first }} \\
\text { / lead time(days) }\end{array}$ & $\begin{array}{c}T V_{10 m} \\
\text { / lead time(days) }\end{array}$ & $\begin{array}{c}N H K_{\text {first }} \\
\text { / lead time(days) }\end{array}$ & $\begin{array}{c}N H K_{5 m} \\
\text { / lead time(days) }\end{array}$ \\
\hline KuToo & Jan 25 & Feb 25 / 31 & Mar $8 / 43$ & Mar $8 / 43$ & Jun $3 / 130$ \\
\hline Cherry blossom party & Apr 9 & Apr $14 / 5$ & Nov $11 / 216$ & Nov $11 / 216$ & Nov $13 / 218$ \\
\hline Image download illegalization & Jan 24 & Jan $7 / 0$ & Feb 14 / 21 & Jan $6 / 0$ & Mar 13 / 49 \\
\hline Part-timer's prank videos & Jan 29 & Jan $30 / 1$ & Feb $6 / 8$ & Feb $6 / 8$ & Feb $13 / 15$ \\
\hline SPA! controversial article & Jan 5 & Jan 8 / 3 & Jan $8 / 3$ & Jan $9 / 4$ & Jan $10 / 5$ \\
\hline Kaneka paternity harassment & Jun 1 & Jun 7 / 6 & Jun $13 / 12$ & $-1-$ & $-1-$ \\
\hline Aichi Triennale & Aug 1 & Aug $2 / 1$ & Aug $3 / 2$ & Aug $3 / 2$ & Aug $3 / 2$ \\
\hline Average lead time & - & 3.20 & 17.20 & 14.0 & 49.75 \\
\hline
\end{tabular}

the party on April 14, but the content of the TV program merely included the people who were invited such as the idol groups; it was not controversial at this time. This issue started receiving criticism in May. However, TV stations began to broadcast this issue after it was revealed that the prime minister invited an unsuitable person to the party on November 8 . This explains the significant time lag between $T V_{\text {first }}$ and $T V_{10 m}, N H K_{\text {first }}, N H K_{5 m}$. The cumulative claims-making frequency of the term "cherry blossom party" is shown in Fig. 2 (b). We noted three sudden increases in the claims-making frequency. The first occurred in early February; however, the proposed method was unable to extract this issue at this time. The second occurred in the middle of April. The proposed method extracted the issue at this time. The third occurred in the middle of May when criticisms began in the Parliament. One of the characteristics of this issue is that most of the claims-making tweets were posted by Cluster 13 (right wing) who oppose to the current Japanese government.

"Image download illegalization" was the only case that the proposed method could not extract earlier than the first TV coverage. This issue began when the Parliament started to revise the copyright law on January 7. All TV stations broadcasted the news of revision at the same time. However, the TV programs merely presented the fact that image download would be prohibited; they did not raise any problematic points at this time. The problematic point of this revision was recognized by the opposition gatherings of the Japan Cartoon Society on February 8. TV stations broadcasted this issue again on February 14 from the perspective of opponents. Subsequently, the Japanese government withdrew the prohibition on March 13. In contrast, the proposed method could extract this issue as early as January 24, earlier than the opposition gathering on February 8 . Therefore, it is safe to say that the proposed method was able to extract this issue earlier than it was actually recognized as a problem. The cumulative claims-making frequency of the term "image download illegalization" is shown in Fig. 2 (c). We noted three sudden increases in the claims-making frequency. The first was observed in late January; the proposed method extracted this issue at this point. The second was in the middle of February, and this corresponded to the opposition gatherings. The third was in early March, and this corresponded with the withdrawal of the prohibition. Compared to other issues, this issue was actively reacted to by Cluster 0 (consumer game) and Cluster 19 (anime geek). Considering the examples of "cherry blossom parties" and "image 
Figure 2: Cumulative frequency of each social issue word in claims-making tweets. The cumulative frequencies are portrayed for each user cluster and it can be observed how each cluster responds to different social issues. Solid lines denote the day when proposed method extracted the issue and dotted lines represent the first TV coverage day. It can be observed that Twitter users react to different social issues based on their interests.

(a)

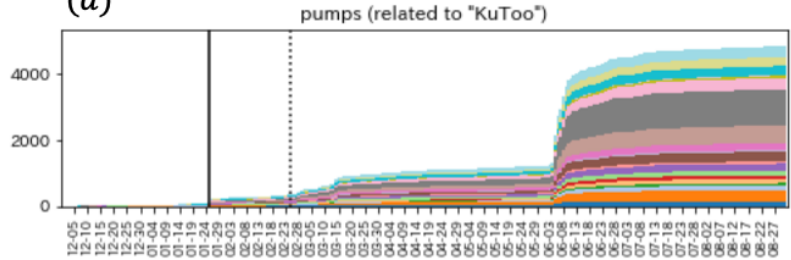

(b)

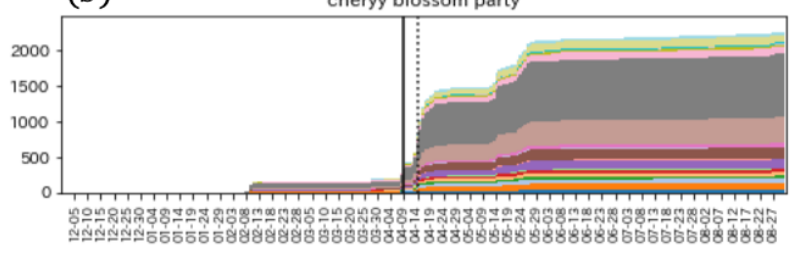

(c)

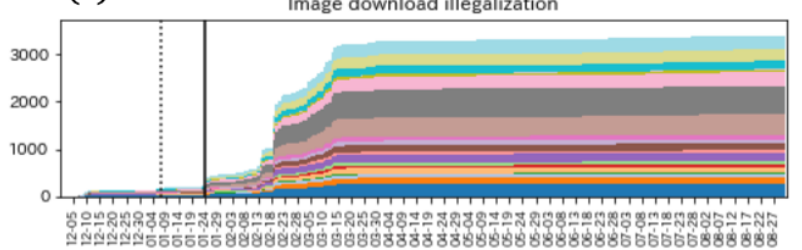

(d)

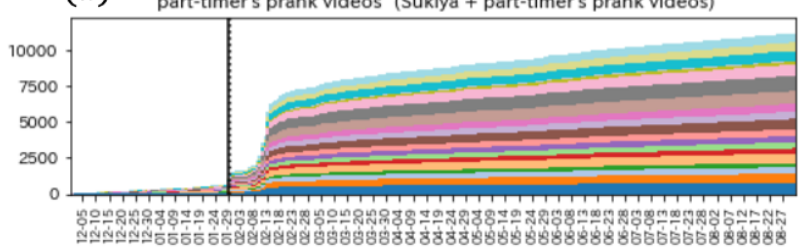

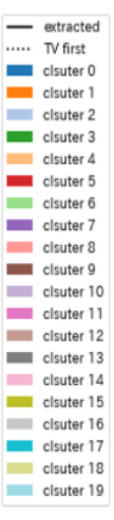

download illegalization," it is evident that Twitter users react to different social issues based on their interests.

One of the applications of this study includes helping policymakers in taking preventive measures for various social issues at the earliest. Policymakers have limited knowledge about the society and our method can extract diverse social issues based on the extraction of social issue-related words and co-occurrence of words with them. Furthermore, our method can also determine the groups of people interested in specific social issues. This information is also helpful for considering the initial responses and taking suitable preventive measures.

The limitation of our methods are as follows. It is difficult for the proposed method to extract those issues that are popular among many people in the early stage. "Part-timer's prank videos" is a good example. This issue happened to Sukiya on January 29 and Kurazushi on February 6. Our method was able to extract this issue at the same time as the Sukiya case, but there was only one day between the extracted day and the day when TV stations first broadcasted this news. Whether this issue is of interest to many people can be verified in Fig. 2 (d). It can be observed that the claimsmaking frequency of this issue is not biased per cluster, unlike other issues. TV stations tend to give priority to the topics that are of interest to many people; therefore, it is reasonable that the proposed method faces difficulty in the early extraction of issues that many people are interested in.

\section{RELATED WORKS}

Most previous researches that attempted to extract social issues aimed to provide an overview of the social problems and applied text mining techniques to news channels and newspapers. Hashimoto et al. extracted articles related to incidents or accidents from newspapers and applied hierarchical clustering to analyze the social issues by selecting important clusters [9]. Jeong et al. extracted sentences that explained the social issues by using topic modeling to Korean newspapers and news channels [15]. While many previous studies used newspapers and news data, Suh focused on the fact that articles on world events circulate over the Internet and proposed a method to extract keywords related to social issues from web news [30]. In comparison to these study, our study aimed at the early extraction of viral spreading social issues and proposed the use of Twitter because news channels and newspapers are not the epicenters of viral spreading social issues. Furthermore, we introduced a novel approach based on the social issues research approach, called constructivism and demonstrated the epoch-making direction for the future research of social issue extraction.

Our work is closely related to several applications of burst detection methods $[8,10,17,18]$ to Twitter streams. Such applications include disaster prevention and aid [26], infectious diseases tracking [6], political topic detection [25], and emerging defect surveillance [35]. Burst detection has been intensively applied for event detection on Twitter [19, 33, 34]. In our study, we employed the $3 \sigma$ burst detection method, which is straightforward and computationally efficient, because we tackled a massive Twitter dataset consisting of tens of billions of tweets. However, our framework is flexible enough to integrate with other burst detection approaches. The recent advances in the burst and event detection methods can also improve our task of the early extraction of viral spreading social issues from Twitter.

Our experimental results showed that it is crucial to preprocess the tweet data for classifying the claims-making tweets and clustering users. Yin et al. also argued that the preprocessing methods for tweet classification and clustering are important for their task of identifying emergency situations on Twitter [36]. It is noteworthy that our results, despite exploring a different domain of the identification of social issues, are consistent with the findings of existing studies. 


\section{CONCLUSIONS}

In this study, we proposed a constructive method for the early extraction of viral spreading social issues. In our method, we first extracted claims-making tweets from Twitter using a supervised machine learning approach. Correspondingly, we prepared training data wherein each tweet was classified into claims-making and non-claims-making tweet. We trained a classifier to identify the claims-making tweets and obtained a high-precision classifier. Subsequently, we identified keywords that were related to new social issues by measuring how their frequencies in the claims-making tweets and their diversity in clusters of social media users. Experiments with large Twitter data showed that the proposed method can successfully extract the social issues by identifying claimsmaking tweets and Twitter-user clusters. Furthermore, on average, the proposed method could extract six out of the seven cases of viral spreading social issues earlier than their first TV coverage, and two weeks earlier than their first national TV coverage. For our future work, we will attempt to create a grouping method for the extracted words, such as the co-occurrence of words, to obtain more comprehensible extraction results.

\section{ACKNOWLEDGMENTS}

This study was supported by a Grant-in-Aid for Young Scientists, Grant Numbers 19K20412, 19K20413.

\section{REFERENCES}

[1] Apoorv Agarwal, Boyi Xie, Ilia Vovsha, Owen Rambow, and Rebecca J Passonneau 2011. Sentiment analysis of twitter data. In Proceedings of the 1st ACL workshop on language in social media. 30-38.

[2] Eiji Aramaki, Sachiko Maskawa, and Mizuki Morita. 2011. Twitter catches the flu: detecting influenza epidemics using Twitter. In Proceedings of the 2011 conference on empirical methods in natural language processing. 1568-1576.

[3] Mario Cataldi, Luigi Di Caro, and Claudio Schifanella. 2010. Emerging Topic Detection on Twitter Based on Temporal and Social Terms Evaluation. In Proceedings of the Tenth International Workshop on MDM.

[4] Tianqi Chen and Carlos Guestrin. 2016. Xgboost: A scalable tree boosting system. In Proceedings of the 22nd acm sigkdd international conference on knowledge discovery and data mining. 785-794.

[5] Yu-Liang Chi. 2009. A consumer-centric design approach to develop comprehensive knowledge-based systems for keyword discovery. Expert Systems with Applications 36 (03 2009), 2481-2493.

[6] Ernesto Diaz-Aviles and Avaré Stewart. 2012. Tracking twitter for epidemic intelligence: case study: Ehec/hus outbreak in germany, 2011. In Proceedings of the 4th annual ACM web science conference. 82-85.

[7] Richard C Fuller and Myers Richard R. 1941. Some Aspects of a Theory of Social Problems. American Sociological Review 6, 1 (1941), 24-32.

[8] Gabriel Pui Cheong Fung, Jeffrey Xu Yu, Philip S Yu, and Hongjun Lu. 2005 Parameter free bursty events detection in text streams. In Proceedings of the 31st international conference on Very large data bases. 181-192.

[9] Taiichi Hashimoto, Koji Murakami, Takashi Inui, Kazuo Utsumi, and Masamich Ishikawa. 2008. Topic Extraction and Social problem detection based on Document Clustering. Fournal of Social Technology Research 5 (2008), 216-226.

[10] Dan He and D Stott Parker. 2010. Topic dynamics: an alternative model of bursts in streams of topics. In Proceedings of the 16th ACM SIGKDD international conference on Knowledge discovery and data mining. 443-452.

[11] Sepp Hochreiter and Jürgen Schmidhuber. 1997. Long Short-term Memory. Neural computation 9 (12 1997), 1735-80.

[12] Philip N Howard, Aiden Duffy, Deen Freelon, Muzammil M Hussain, Will Mari, and Marwa Maziad. 2011. Opening closed regimes: what was the role of social media during the Arab Spring? Available at SSRN 2595096 (2011).

[13] JAPAN PRESS RESEARCH INSTITUTE. 2019. Summary of the 12th National Opinion Poll on Media (2019). Technical Report. https://www.chosakai.gr.jp/ project/notification/

[14] Shiau Chou Jen, Masano Ochi, Takeshi Sakaki, Junichirou Mori, and Ichirou Sakata. 2019. Building a dataset with crowdsourcing for early social issues extraction. ICS 2019-ICS-196, 9 (2019), 1-5.
[15] Dami Jeong, Jaeseok Kim, Gi-Nam Kim, Jong-Uk Heo, Byung-Won On, and Mijung Kang. 2013. A Proposal of a Keyword Extraction System for Detecting Social Issues. Fournal of Intelligence and Information Systems 19 (09 2013).

[16] Yoon Kim. 2014. Convolutional Neural Networks for Sentence Classification. In Proceedings of the 2014 Conference on Empirical Methods in Natural Language Processing (EMNLP). 1746-1751.

[17] Jon Kleingberg. 2002. Bursty and hierarchical structure in streams. Proc. 8th ACM SIGKDD International Conference on Knowledge Discovery and Data Mining, Edmonton, Alberta, Canada, fuly 2002 (2002), 1-25.

[18] Jure Leskovec, Andreas Krause, Carlos Guestrin, Christos Faloutsos, Jeanne VanBriesen, and Natalie Glance. 2007. Cost-effective outbreak detection in networks. In Proceedings of the 13th ACM SIGKDD international conference on Knowledge discovery and data mining. 420-429.

[19] Chenliang Li, Aixin Sun, and Anwitaman Datta. 2012. Twevent: segment-based event detection from tweets. In Proceedings of the 21st ACM international conference on Information and knowledge management. 155-164.

[20] Shogo Matsuno, Sakae Mizuki, and Takeshi Sakaki. 2019. Constructing of the word embedding model by Japanese large scale SNS + Web corpus. Proceedings of the Annual Conference of JSAI JSAI2019 (2019).

[21] Tomas Mikolov, Ilya Sutskever, Kai Chen, Greg S Corrado, and Jeff Dean. 2013. Distributed representations of words and phrases and their compositionality. In Advances in neural information processing systems. 3111-3119.

[22] Yuhiro Mizunuma, Shuhei Yamamoto, Yutaro Yamaguchi, Atsushi Ikeuchi, Tetsuji Satoh, and Satoshi Shimada. 2014. Twitter bursts: Analysis of their occurrences and classifications. Proceedings of the 8th international conference on digital society (2014), 182-187.

[23] Tetsuya Nasukawa and Jeonghee Yi. 2003. Sentiment analysis: Capturing favorability using natural language processing. In Proceedings of the 2nd international conference on Knowledge capture. 70-77.

[24] Jonathon Read. 2005. Using emoticons to reduce dependency in machine learning techniques for sentiment classification. In Proceedings of the ACL student research workshop. 43-48.

[25] Sven Rill, Dirk Reinel, Jörg Scheidt, and Roberto V Zicari. 2014. Politwi: Early detection of emerging political topics on twitter and the impact on concept-level sentiment analysis. Knowledge-Based Systems 69 (2014), 24-33.

[26] Takeshi Sakaki, Makoto Okazaki, and Yutaka Matsuo. 2010. Earthquake shakes Twitter users: real-time event detection by social sensors. In Proceedings of the 19th international conference on world wide web. 851-860.

[27] Jagan Sankaranarayanan, Hanan Samet, Benjamin E Teitler, Michael D Lieberman, and Jon Sperling. 2009. Twitterstand: news in tweets. In Proceedings of the 17th ACM sigspatial international conference on advances in geographic information systems. $42-51$.

[28] Malcolm Spector and John I Kitsuse. 1977. Constructing social problems. Menlo Park, Calif. : Cummings Pub. Co.

[29] Dario Stojanovski, Gjorgji Strezoski, Gjorgji Madjarov, and Ivica Dimitrovski. 2016. Finki at semeval-2016 task 4: Deep learning architecture for twitter sentiment analysis. In Proceedings of the 10th international workshop on semantic evaluation. $149-154$.

[30] Jong Suh. 2019. SocialTERM-Extractor: Identifying and Predicting Social-ProblemSpecific Key Noun Terms from a Large Number of Online News Articles Using Text Mining and Machine Learning Techniques. Sustainability 11 (01 2019), 196.

[31] Kazuo Utsumi, Takashi Inui, Taiichi Hashimoto, Koji Murakami, and Masamichi Ishikawa. 2009. Extraction of Critical Knowledge Concerning Socila Problems and Technological Solutions. Journal of Social Technology Research 6 (2009), 187-198.

[32] Xin Wang, Yuanchao Liu, Chengjie Sun, Baoxun Wang, and Xiaolong Wang. 2015. Predicting Polarities of Tweets by Composing Word Embeddings with Long Short-Term Memory. In Proceedings of the 53rd Annual Meeting of the Association for Computational Linguistics and the 7th International foint Conference on Natural Language Processing (Volume 1: Long Papers). 1343-1353.

[33] Jianshu Weng and Bu-Sung Lee. 2011. Event detection in twitter. In Proceedings of the 5th international AAAI conference on weblogs and social media.

[34] Wei Xie, Feida Zhu, Jing Jiang, Ee-Peng Lim, and Ke Wang. 2016. Topicsketch: Real-time bursty topic detection from twitter. IEEE Transactions on Knowledge and Data Engineering 28, 8 (2016), 2216-2229.

[35] Jiejun Xu, Daniel Xie, Tsai-Ching Lu, and John Cafeo. 2017. EDSV: Emerging Defect Surveillance for Vehicles. In Proceedings of the 9th ACM on web science conference. 219-222.

[36] Jie Yin, Sarvnaz Karimi, Andrew Lampert, Mark Cameron, Bella Robinson, and Robert Power. 2015. Using social media to enhance emergency situation awareness. In Proceedings of the 24th international joint conference on artificial intelligence. 